\title{
Erratum to: Pareto Optimal Scheduling of Synchronous Data Flow Graphs via Parallel Methods
}

\author{
Yu-Lei Gu ${ }^{1,2(\bowtie)}$, Xue-Yang $\mathrm{Zhu}^{2}$, and Guangquan Zhang ${ }^{1}$ \\ 1 State Key Laboratory of Computer Science, Institute of Software, Chinese \\ Academy of Sciences, Beijing, China \\ \{guyl, zxy\}@ios.ac.cn \\ 2 School of Computer Science and Technology, Soochow University, Suzhou, China \\ gqzhang@suda.edu.cn
}

\section{Erratum to: \\ Chapter 14 in: X. Li et al. (Eds.) Dependable Software Engineering DOI: $10.1007 / 978-3-319-25942-0 \_14$}

In the original version, the affiliations of the second and third author are incorrect.

Instead of Xue-Yang $\mathrm{Zhu}^{2}$ and Guangquan $\mathrm{Zhang}^{1}$ it should be read as Xue-Yang Zhu ${ }^{1}$ and Guangquan Zhang ${ }^{2}$.

The online version of the original chapter can be found under

DOI: 10.1007/978-3-319-25942-0_14

(C) Springer International Publishing Switzerland 2015

X. Li et al. (Eds.): SETTA 2015, LNCS 9409, p. E1, 2015.

DOI: $10.1007 / 978-3-319-25942-0 \_21$ 